\title{
Database on pharmacophore analysis of active principles, from medicinal plants
}

\author{
Daisy Pitchai*, Rajalakshmi Manikkam, Sasikala R. Rajendran, Gnanamani \\ Pitchai
}

Bioinformatics centre (BIF), PG, Research Department of Biotechnology, Bioinformatics,Holy Cross College(Autonomous), Teppakulam, Tiruchirapalli620002, Tamilnadu, India; daisy pitchai-E mail:daisylesslie@gmail.com; Fax: 0431 2713312; *Corresponding Author

Received January 20, 2010; revised April 7, 2010; accepted June 8, 2010; published July 6, 2010

\begin{abstract}
:
Plants continue to be a major source of medicines, as they have been throughout human history. In the present days, drug discovery from plants involves a multidisciplinary approach combining ethnobotanical, phytochemical and biological techniques to provide us new chemical compounds (lead molecules) for the development of drugs against various pharmacological targets, including cancer, diabetes and its secondary complications. In view of this need in current drug discovery from medicinal plants, here we describe another web database containing the information of pharmacophore analysis of active principles possessing antidiabetic, antimicrobial, anticancerous and antioxidant properties from medicinal plants. The database provides the botanical, taxonomic classification, biochemical as well as pharmacological properties of medicinal plants. Data on antidiabetic, antimicrobial, anti oxidative, anti tumor and anti inflammatory compounds, and their physicochemical properties, SMILES Notation, Lipinski’s properties are included in our database. One of the proposed features in the database is the predicted ADMET values and the interaction of bioactive compounds to the target protein. The database alphabetically lists the compound name and also provides tabs separating for anti microbial, antitumor, antidiabetic, and antioxidative compounds.
\end{abstract}

Availability: http://www.hccbif.info /

Keywords: medicinal plants database; anti-diabetic compounds; antioxidant compounds; antimicrobial compounds; anticarcinogenic compounds; ADMET properties: pharmacophore analysis

\section{Background:}

Plants form the main ingredients of medicines in traditional systems of healing and have been the source of inspiration for several major pharmaceutical drugs. The use of plants as medicines has involved the isolation of active compounds, beginning with the isolation of morphine from opium in the early 19th century [1] and subsequently led to the isolation of early drugs such as cocaine, codeine, digitoxin and quinine, of which some are still in use [2, 3]. Isolation and characterization of pharmacologically active compounds from medicinal plants continue today. Investigation into the chemical composition of medicinal plants and especially secondary metabolites is a dynamic research field worldwide and is the base for Drug discovery. There is an increasing demand to identify and analyze the target proteins with their active sites and potential drug molecules that can bind to these sites specifically.Many databases are available today to describe the medicinal plants and compounds $[4,5,6]$. Most of the documentation in those databases is focused on the basic information about the plants and compounds and their targets. Our focus is to provide the complete pharmacophore analysis of active principles possessing antidiabetic, antimicrobial, anticancerous and antioxidant properties from medicinal plants and the interaction of the compounds with their target proteins. So as an additional effort to decipher research into technology this data base is created to document the complete research literatures about the medicinal compounds and the active site of their target proteins available and docked results of the compounds and their target proteins.

\section{Methodology:}

Data collection:

Scientific literatures related to the medicinal plants were collected using Pubmed (NCBI) [7], ScienceDirect [8], Biomed Central [9], Springerlink
[10], Scirus [11] Wiley journals [12]. Databases such as Floridata [13], Plants for A Future database [14] and Germplasm Resource Information Network [15] were also used to retrieve information about the plants. The database includes 163 active compounds having antidiabetic (32 compounds), anti-cancerous (83compounds), antimicrobial (43 compounds) and anti-oxidative (5 compounds) effects. The databases are organized in a hierarchical classification that simplifies the process of finding the active principles having a particular effect.

\section{Ligand and target analysis for docking:}

Physicochemical and chemical properties of the compounds were calculated using chemsketch tool and cross-checked with pubchem data entries. Two dimensional structure was drawn using Arguslab. ADMET properties of the active principles were predicted using Accord for Excel 6.1, an accelry's product. It tends to focus on application of plant drugs, their properties and their ADMET (Absorption, Distribution, Metabolism, Excretion and Toxicity)nature. The three dimensional structure of the proteins were retrieved from protein Data Bank (PDB) which is a repository for the processing and distribution of 3D- structure data of large molecules of proteins and nucleic acids. The docking analysis of the compounds was carried out by Libdock of Discovery studio 2.3 Version (Accelrys Software Inc).

\section{Content organization:}

Based on the biological activity the data documentation is organized in the database. Each section contains a list of active principle index and with five pages for each active principle. The first page provides brief descriptions of the plant source including its taxonomic classification, common name, other active principles, distribution and its medicinal use with appropriate references. The second page includes data regarding 


\section{Bioinformation}

active principles, its physicochemical properties, mechanism of action with its pubmed ID,2D structure of the active principles. The third page lists the ADMET values of active principles includes solubility, intestinal absorption, blood brain barrier penetration level, inhibiton on Cytochrome $\mathrm{P}_{450}$ enzyme, carrier protein binding level, hepatoxicity values and the key notes for interpreting the predicted values. Protein target name, synonym, sequence length, PDB structure ID, domain and the Sequence in FASTA format are given in the fourth page. Protein-Ligand interactions details including their binding site, pose number, grid volume, Libdock score, contact information (van der Waals radii) and their docked complex image are presented in the fifth page.

\section{Conclusion:}

In the current research scenario there is an increasing demand for the data regarding the active principles with their target sites. The database provides the botanical, taxonomic classification, biochemical as well as pharmacological properties of medicinal plants. Data on anti-diabetic, antimicrobial, anti oxidative, anti tumor and anti inflammatory compounds, and their physicochemical properties, SMILES Notation, Lipinski's properties are included in our database. One of the proposed features in the database is the predicted ADMET values and the interaction of bioactive compounds to the target protein. We hope that this data base will provide complete details about most potent drug like molecules for diabetes, microbial infections, cancer and oxidative stress. This database finds utility to the scientific community for a quick review on bioactive compounds for drug development research and provides enormous scope for development of drugs.

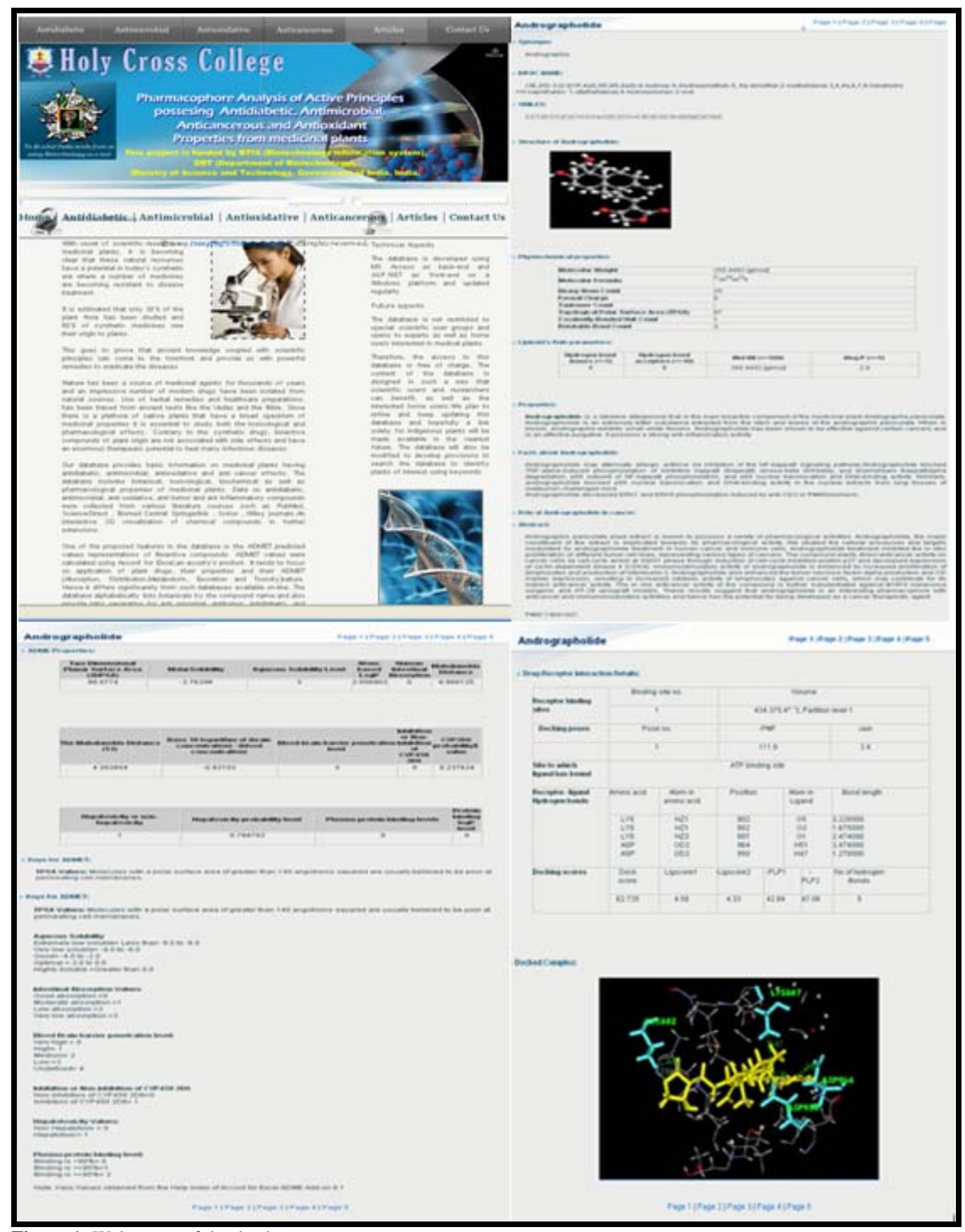

Figure 1: Web pages of the database 
Future development:

We plan to refine and keep updating this database and hopefully a link solely for indigenous plants will be made available in the nearest future. The database will also be modified to develop provisions to search the database to identify plants of interest using keywords.

\section{Acknowledgement:}

This project is funded by BTIS (Biotechnology information system), DBT (Department of Biotechnology), Ministry of Science and Technology, Government of India, India.

\section{References:}

[1] AD Kinghorn, J Pharm Pharmacol (2001) 53: 135.

[2] DJ Newman et al. Nat Prod Rep (2000) 17: 215.
[3] MS Butler, J Nat Prod (2004) 67: 2141.

[4] http://www.wam.umd.edu/ mct/Plants/medicinal.html

[5] http://www.ars-grin.gov/duke/

[6] http://hccbif.org/

[7] http://www.ncbi.nlm.nih.gov/entrez/query.fcgi?d b=PubMed

[8] http://www.sciencedirect.com

[9] http://www.biomedcentral.com

[10] http://www.springerlink.com

[11] http://www.scirus.com

[12] http://www3.interscience.wiley.com/cgibin/home

[13] http://www.floridata.com/ref/A/asim_tri.cfm

[14] http://www.pfaf.org/index.php- Plants For A Future

[15] http://www.ars-grin.gov/ 